\title{
Increasing prevalence of Addison's disease in German females: health insurance data 2008-2012
}

\author{
Gesine Meyer, Kathrin Neumann', Klaus Badenhoop and Roland Linder ${ }^{1}$ \\ Division of Endocrinology, Department of Medicine 1, University Hospital, Goethe-University Frankfurt, \\ Theodor-Stern-Kai 7, D-60590 Frankfurt, Germany and ${ }^{1}$ WINEG Scientific Institute of the TK for Benefit and \\ Efficiency in Health Care, Hamburg, Germany
}

Correspondence should be addressed to $\mathrm{G}$ Meyer Email Gesine.Meyer@kgu.de

\begin{abstract}
Objective: Our objective was to investigate the epidemiology of autoimmune Addison's disease (AD) in Germany. Design: Routine data were analyzed from the Statutory Health Insurance (SHI) database of the Techniker Krankenkasse (TK) for an observation period from 01/01/2008 to 31/12/2012. The TK is one of the largest German health care insurance providers covering more than $10 \%$ of the German population.

Subjects and methods: Between 2008 and 2012, a total of 2477 diagnoses of primary adrenal failure were recorded in the SHI database. After exclusion of secondary, iatrogenic or other non-idiopathic forms and after adjustment for incomplete data sets, 1364 diagnoses of autoimmune-mediated $A D$ remained.

Results: The prevalence of $A D$ in our cohort showed a steady increase from 82 per million in 2008 to 87 per million in 2012. On average, the prevalence rose about $1.8 \%$ per year, and due to a pronounced increase $(2.7 \%)$ in females. The prevalence was lower in men (63-68 per million) than in women (96-108 per million). Autoimmune comorbidities were found in $46.5 \%$ of $A D$ patients. Adrenal crises were documented with a frequency of 14-17/100 patient years.

Conclusions: These data provide a first epidemiological profile of this rare and perilous endocrine disease in Germany. Although the prevalence of $A D$ appears lower than in the Scandinavian countries, the increasing figures in females over the last 5 years warrant further investigations. Furthermore, adrenal crises pose a considerable burden. Hereby, we can show that health insurance data provide a valuable tool for epidemiological studies in the absence of national registries.
\end{abstract}

\section{Introduction}

Addison's disease (AD) is a chronic disease leading to a deficient production of glucocorticoids, mineralocorticoids, and androgens in the adrenal cortex. The disease is rare and valid epidemiological data are difficult to collect. For the European countries, the prevalence is estimated at 93-144 cases per million $(1,2)$. These estimations, however, are based on few data from rather small populations - less than one million - in single European regions $(3,4,5,6)$. Most convincing data come from the Scandinavian countries, where national patient registries exist $(7,8)$. Norway and Sweden, moreover, have the highest prevalence for autoimmune AD with 144 and 136 per million respectively $(6,7,8)$. Primary adrenal insufficiency occurs more frequently in women compared with men with an odds ratio around 1.2 for women $(1,5)$.

The incidence of $\mathrm{AD}$ in Europe is estimated at 4.4-6.2 per million population per year. Several recent studies have pointed to an increasing incidence of the disease in the last decades $(1,2,4,6)$. Very recently, a Swedish study, based on the Swedish National Prescribed Drug Register, has shown a distinct increase in yearly prevalence for autoimmune AD from 12.2 to 13.1/100 000 person years in the period 2005-2009 (8). Considering the continuous decline in tuberculous adrenalitis, one of the major causes

Published by Bioscientifica Ltd. 
for $\mathrm{AD}$ in the past, other causes of this idiopathic adrenal failure prevail. Nowadays, in developed countries, autoimmune-mediated primary adrenal insufficiency accounts for more than $80 \%$ of cases (2). Therefore, a likely explanation for the increasing incidence of $\mathrm{AD}$ is a broad progress of autoimmunity in general.

In patients with autoimmune-mediated AD, autoimmune comorbidity is rather frequent. Approximately $60 \%$ of these patients develop an autoimmune polyglandular syndrome (APS) comprising autoimmune AD and at least one other autoimmune disease like autoimmune thyroid disease, type 1 diabetes mellitus, autoimmune oophoritis leading to primary ovarian failure, celiac disease, atrophic gastritis, vitiligo, and/or autoimmune alopecia $(8,9)$.

Although Germany is the most populous country in Europe, there are no specific national epidemiological data about AD so far. The primary aim of this study was to obtain reliable epidemiological data for rare autoimmune $\mathrm{AD}$ in Germany in a large cohort with a focus on the overall prevalence, changes in prevalence during the observation period, and gender differences as well as the occurrence of Addison crises. Secondary aims were to retrieve data of associated autoimmune diseases, their frequency and the relative risk for Addison patients to develop further components of APS.

For this purpose, between 2008 and 2012, the Statutory Health Insurance (SHI) database of the Techniker Krankenkasse (TK) was analyzed. The TK is one of the large German health insurance providers, covering more than $10 \%$ of the population. Their members are representative for the country with a slight tendency toward more skilled professions requiring a higher education and to male insurants with a gender ratio of 1.04 (male:female) compared with 0.96 in the general population. Germany was the first nation worldwide to introduce a SHI system in 1883. For everybody, it is obligatory to be medically insured and SHI institutions, such as the TK, cover around $90 \%$ of the population. The registration with a SHI is independent of age, sex, and state of health by law and happens through the work contract of those in a job, alternatively in retirement or through social security if unemployed. Children and husbands/wives of employees are coinsured through the same system. Through the random process of obligatory health insurance, it can be assumed that the proportion of AD patients is comparable in all German statutory health care insurances. Thus, the TK database will detect a representative cohort of $\mathrm{AD}$ in the German population.

\section{Subjects and methods}

The SHI database of the TK provides a documentation of diagnoses by all members with a doctor contact, comprising data of inpatient and ambulatory medical treatment as well as outpatient surgery.

Diagnoses are encoded by the International Statistical Classification of Diseases and Related Health Problems (ICD-10). These data were evaluated by the Scientific Institute Wissenschaftliches Institut der TK für Nutzen und Effizienz im Gesundheitswesen - WINEG.

We analyzed routine data from the TK. ICD-codes as accounting data are transferred in the SHI database automatically. As all data are linked to the patients insurance registration number, they are individual related and not exchangeable. Altogether, in the observation period from $01 / 01 / 2008$ to $31 / 12 / 2012$, more than 30 million men were evaluated each year.

A query for ICD-10 codes E27.1 (primary adrenal failure), E27.2 (adrenal crisis), and E31.0 (APS) was implemented, the latter by filtering for the medications containing hydrocortisone (ATC code H02AB09) and/or fludrocortisone (ATC code H02AA02) in the year of diagnosis. Subsequently, all causes of secondary, iatrogenic, infectious, traumatic, or other non-idiopathic forms of primary adrenal failure were excluded by filter out ICD-10 codes E23.0 (pituitary failure), E22 (pituitary hyperfunction), E24 (Cushing's syndrome), D35.2, D35.3, D44.3, and D44.4 (pituitary tumors), E27.3 (drug-induced adrenal failure), E89.6 (adrenal failure caused by medical measure), A18.7 (tuberculous adrenalitis), A39.1 (Waterhouse-Friderichsen syndrome), E27.8 (miscellaneous disease of adrenal gland), E27.4 (miscellaneous adrenal failure), E25.8, E25.00, E25.01, E25.08, E25.09 (different forms of congenital adrenal hyperplasia), E71.3 (adrenoleucodystrophy), E35.1 (adrenal diseases relating to other classified disorders), Q89.1 (congenital dysplasia of adrenal gland), D44.1 (adrenal tumor), D35.0 (benign adrenal tumor), C79.7 (adrenal metastasis), and C74.9 (malignant adrenal tumor).

Patients with $\mathrm{AD}$, defined in this manner as autoimmune-mediated $\mathrm{AD}$, were screened for associations with other autoimmune diseases, searching for codes K29.4 (atrophic gastritis), K75.4 (autoimmune hepatitis), K90 (celiac disease), M35 (collagen disease), E05 (Graves' disease), E06 (Hashimoto's thyroiditis), E20 (hypoparathyroidism), M32.1/.8/.9 (lupus erythematosus), D51.0 (pernicious anemia), E28.3 (primary ovarian failure), M05/M06 (rheumatoid arthritis), E10 (type 1 diabetes mellitus), and L80 (vitiligo). 
Data were collected annually for the years 2008-2012. All data were adjusted to the general population of Germany for age and sex, using the data of the German Federal Office of Statistics from 2010.

In an analogous manner, we implemented a query for the autoimmune diseases, type 1 diabetes mellitus (ICD-10 code E10) and vitiligo (ICD-10 code L80), in all TK-insurants. These data were collected annually for the years 2009-2011. The study was approved by the Local Ethical Committee.

\section{Results}

Between 2008 and 2012, 2477 diagnoses of primary adrenal failure were recorded in the SHI database. After exclusion of secondary, iatrogenic, or other nonidiopathic forms and after adjustment for incomplete data sets, 1364 diagnoses of autoimmune-mediated AD remained.

Therefore, in our cohort, representing $\sim 10 \%$ of the German population, the prevalence of autoimmunemediated $\mathrm{AD}$ was found to range between 80 and 87 per million. The prevalence showed an increase from 82 per million in 2008 to 87 per million in 2012. On average, the prevalence rose about $1.8 \%$ per year (0.2\% in men and $2.7 \%$ in women; Fig. 1$)$.

Diagnosis was documented in $n=536$ male and $n=828$ female insurants. The percentage of affected males varied between 38.2 and 41.5\%. Altogether, the prevalence of autoimmune $\mathrm{AD}$ was lower in men

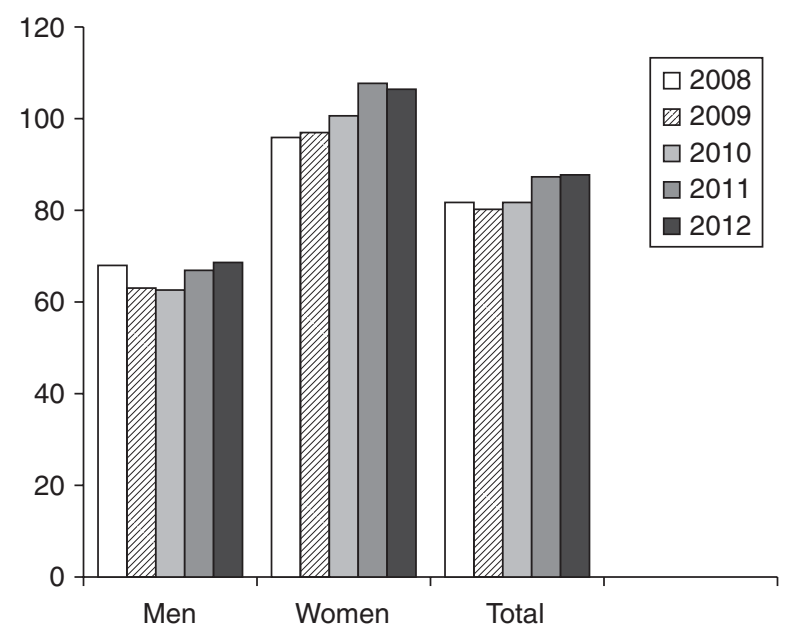

\section{Figure 1}

Prevalence of autoimmune Addison's disease in Germany in 2008-2012 (per million).
(63-68 per million) than in women (96-108 per million) with an odds ratio of 1.6 (95\% CI: 1.3-1.8) for women. Male patients were 5-94 years old (mean age: 51 years) compared with female patients aged 4-95 years (mean age: 48 years). Adrenal crises were documented with a frequency of 14-17/100 patient years.

Autoimmune comorbidities were found in $46.5 \%$ $(n=634)$. Commonest were autoimmune thyroid diseases and type 1 diabetes mellitus, followed by rheumatoid diseases, vitiligo, atrophic gastritis, and primary ovarian failure (Fig. 2). Individual patients had up to five additional autoimmune diseases $(+1,61.4 \%$; $+2,29.1 \%$; $+3,7.3 \%$; $+4,1.9 \%$; and $+5,0.3 \%$ ).

Compared with TK-insurants without $\mathrm{AD}$, patients with $\mathrm{AD}$ were found to have a severe risk for diverse additional autoimmune-mediated diseases of endocrine and non-endocrine organs up to an odds ratio of $>30$. Hereby, autoimmune thyroid diseases not only showed the highest risk, diagnosed in $44.5 \%$, but also type 1 diabetes was found in nearly $12 \%$ of $\mathrm{AD}$ patients. Rare conditions like pernicious anemia, autoimmune hypoparathyroidism, and autoimmune hepatitis illustrate the broad spectrum of autoimmunity in AD patients (Table 1).

To compare potential changes in the prevalence of other autoimmune diseases, we specifically investigated the change in the prevalence of type 1 diabetes mellitus: its prevalence in all TK-insurants lay between 7961 and 8313 per million and did not change significantly over the years 2009-2011. A similar result was seen for vitiligo with a prevalence between 1395 and 1422 per million, with no significant change.

\section{Discussion}

Epidemiological data in $\mathrm{AD}$ have been provided not only by either Scandinavian registries but also through investigator-initiated databases in the academic centers with a large referral area $(3,4,5)$. We provide such data for the first time through a SHI. Such a database implies potential limitations. As coding is the basis for reimbursement both in hospitals and in outpatient care, we assume a correct code for rare AD both by primary care physicians as well as by specialists, such as in endocrinology. However, incomplete diagnosis coding cannot be ruled out particularly in autoimmune polyendocrine symptom (APS) patients. In this study, we filtered out other forms of adrenal failure to minimize this error rate and included only those APS patients with an AD steroid substitution profile. Furthermore, we confined the query for differential diagnoses to the year quarter, in which the diagnosis 


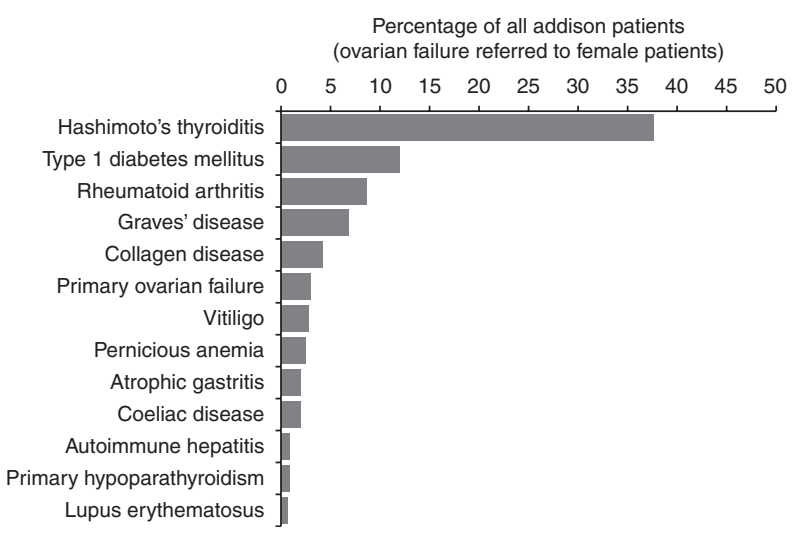

Figure 2

Autoimmune comorbidity in patients with Addison's disease, data in percent of all Addison patients, ovarian failure referred to female patients only.

of AD was encoded. This approach bears a remaining risk for incorrect coding particularly missing some AD patients. We therefore assume a conservative estimation of the true prevalence.

In the SHI database of the TK, patients, and therefore their diagnoses, are recorded only when they had an inpatient or outpatient doctor contact due to this diagnosis in a particular year. For this reason, our results reveal indications for the prevalence of autoimmune AD related to a distinct year and not for the incidence of this disease. As patients with primary adrenal insufficiency depend on a lifelong corticosteroid supplementation therapy and this medication is available only on prescription in Germany, each Addison patient has at least one medical contact per year.

The retrieval system using data from a large statutory health care insurance was valid and comparable to those from other countries with national diagnostic ascertainment such as Sweden and Norway $(6,7,8)$. Thereby this novel tool allows epidemiological studies on rare diseases in the absence of national registries. Moreover, health care providers would benefit by allocating medical resources based on reliable diagnostic data and can improve the long-term management of both rare and more common chronic disorders.

We found a prevalence of autoimmune $\mathrm{AD}$ in Germany between 82 and 87 per million. This prevalence appears lower than in the Scandinavian countries, Norway (144 per million) and Sweden (136 per million) $(6,7)$. It is also lower than most of the estimations for other European countries so far $(3,4,5,6)$. As the latter figures are based on few data from very small populations, their validity is limited. Owing to the large size of the TK-insurants, covering nearly $10 \%$ of the German population, and the

Table 1 Odds ratio (OR) for 13 autoimmune comorbidities in individuals with autoimmune Addison's disease (AD) compared with individuals without $A D$ (controls).

\begin{tabular}{|c|c|c|c|c|c|c|c|}
\hline \multirow[b]{2}{*}{ ICD } & \multicolumn{2}{|c|}{$\begin{array}{l}\text { Individuals with } \\
\text { AD in } 2012(n=712)\end{array}$} & \multicolumn{2}{|c|}{$\begin{array}{c}\text { Controls } \\
(n=8540805) \text { in } 2012\end{array}$} & \multirow[b]{2}{*}{ OR } & \multirow[b]{2}{*}{ Lower $95 \% \mathrm{Cl}$} & \multirow[b]{2}{*}{ Upper $95 \% \mathrm{Cl}$} \\
\hline & With ICD & Without ICD & With ICD & Without ICD & & & \\
\hline $\begin{array}{l}\text { E06.3: Hashimoto's } \\
\text { thyroiditis }\end{array}$ & 268 & 444 & 171663 & 8369142 & 29.4 & 26.7 & 32.5 \\
\hline $\begin{array}{l}\text { E10: type } 1 \text { diabetes } \\
\text { mellitus }\end{array}$ & 85 & 627 & 74506 & 8466299 & 15.4 & 13.0 & 18.2 \\
\hline $\begin{array}{l}\text { M05/M06: rheumatoid } \\
\text { arthritis }\end{array}$ & 61 & 651 & 86372 & 8454433 & 9.2 & 7.4 & 11.4 \\
\hline E05.0: Graves' disease & 49 & 663 & 37003 & 8503802 & 17.0 & 13.7 & 21.0 \\
\hline M35.0: collagen disease & 29 & 683 & 81926 & 8458879 & 4.4 & 3.1 & 6.2 \\
\hline $\begin{array}{l}\text { E28.3: primary ovarian } \\
\text { failure }\end{array}$ & 13 & 699 & 21944 & 8518861 & 7.2 & 4.5 & 11.5 \\
\hline L80: vitiligo & 21 & 691 & 14488 & 8526317 & 17.9 & 13.1 & 24.5 \\
\hline $\begin{array}{l}\text { D51.0: pernicious } \\
\text { anemia }\end{array}$ & 18 & 694 & 6773 & 8534032 & 32.7 & 24.3 & 43.9 \\
\hline K29.4: atrophic gastritis & 14 & 698 & 6919 & 8533886 & 24.7 & 17.3 & 35.3 \\
\hline K90.0: celiac disease & 14 & 698 & 11730 & 8529075 & 14.6 & 9.8 & 21.7 \\
\hline $\begin{array}{l}\text { K75.4: autoimmune } \\
\text { hepatitis }\end{array}$ & 7 & 705 & 2453 & 8538352 & 34.6 & 21.8 & 54.9 \\
\hline $\begin{array}{l}\text { E20: primary hypo- } \\
\text { parathyroidism }\end{array}$ & 7 & 705 & 3094 & 8537711 & 27.4 & 16.8 & 44.7 \\
\hline $\begin{array}{l}\text { M32.1/M32.8/M32.9: } \\
\text { lupus erythematosus }\end{array}$ & 5 & 707 & 4090 & 8536715 & 14.8 & 7.6 & 28.6 \\
\hline
\end{tabular}

www.eje-online.org 
quantity of analyzed data, we assume that our findings are sufficiently valid. Our data provide a first epidemiological profile of rare and perilous AD in Germany.

In our cohort, adrenal crises were documented with a frequency of 14-17/100 patient-years. This finding exceeds previous observations considerably. Former data of a German population showed about 6.3-crises/100 patient-years calculated by patient questionnaires in a mixed cohort of patients with primary and secondary adrenal insufficiency (10). In agreement with existing data, $\mathrm{AD}$ affects more frequently women than men in our cohort with an odds ratio slightly higher than the reported one (1.6 vs 1.2 ).

Furthermore, we observed an annual increase in prevalence of about $1.8 \%$ per year on average. A relevant change in the medical profession's awareness over the years 2008-2012 for AD as reason for this observation is not apparent. Actually, the awareness is still insufficient. Two recent studies have shown a delay of diagnosis $(11,12)$. As medical treatment of AD has not changed over decades, we assume that there is no change in life expectancy beyond that of the general population.

Remarkably, the increment of prevalence was nearly exclusively seen in female individuals $(2.7 \%$ per year in average vs $0.1 \%$ in males). To the best of our knowledge, this is the first study of AD that showed a difference in the increasing prevalence for women and men.

An increasing incidence has been observed in $\mathrm{AD}(1,2$, $4,6,8)$ and several other autoimmune-mediated disorders in recent years, including celiac disease (13), type 1 diabetes $(14,15,16,17,18)$, and multiple sclerosis (19). Diverse genetic variations predispose to autoimmune $\mathrm{AD}$ $(20,21,22)$. Most of them are susceptibility factors for autoimmunity in general. However, genetic susceptibility variants are rather frequent in the general population, and their role for an increase in prevalence of autoimmune diseases is probably not relevant because their frequencies have not changed. More likely is the role of environmental factors to cause and/or trigger organ-specific autoimmunity. In recent years, vitamin D deficiency has been implicated as a potential environmental factor for autoimmune disorders $(23,24)$. We have no data about a possible change in prevalence of vitamin $\mathrm{D}$ deficiency in AD patients during the study period, but in patients with type 1 diabetes we do observe such a trend (25). Further investigations in patients with $\mathrm{AD}$ are necessary.

Other environmental agents, such as viral infections, cigaret smoking, and pollutants, as well as a reduced microbial burden during early life, have been found to influence the development of autoimmune diseases, presumably by epigenetic changes $(26,27,28)$.

A striking finding is that particularly females show an increasing prevalence over the last 5 years. One possible explanation is a growing trend for autoimmunity in general. However, in contrast to preceding studies $(14,15,16,17,18)$, in our cohort the prevalence data for the much more frequent autoimmune diseases type 1 diabetes mellitus and vitiligo did not change relevantly over the years 2009-2011, but the triggering and/or causative agents in rare $\mathrm{AD}$ may differ from those more common disorders.

Possibly, so far undetected environmental factors may underlie the distinct rise of autoimmune $\mathrm{AD}$ in the recent years. The stronger increase in prevalence in women raises the question, which environmental factors may affect females more than males. A stronger increase in type 1 diabetes has recently been described in Sardinian girls who might be more vulnerable to environmental factors (13).

In patients with autoimmune-mediated $\mathrm{AD}$, autoimmune comorbidity is rather frequent and occurs in $\sim 60 \%$ of patients. In our cohort, the rate was lower with an autoimmune comorbidity of $46.5 \%$. Conceivably, focusing on the principal diagnosis of $\mathrm{AD}$, comorbidities may not have been coded for in every case. Furthermore, analysis of data was limited to the years 2008-2012, so future manifestations of autoimmune-mediated diseases in patients with $\mathrm{AD}$ could not be regarded. This would lead to an underestimation especially of autoimmune comorbidity, which is possibly more frequent than we observe.

We found a severe risk for diverse additional autoimmune-mediated diseases of endocrine and nonendocrine organs in patients with autoimmune AD up to an odds ratio of $>30$ compared with individuals without $\mathrm{AD}$. This impact was expectedly strong for frequent autoimmune diseases like Hashimoto's thyroiditis (odds ratio 29.4), but notably also for rare disorders like pernicious anemia (odds ratio 32.7), autoimmune hepatitis (odds ratio 34.6), and atrophic gastritis (odd ratio 24.7).

Our cohort is confined to German health care insurants. Further research in other countries is necessary to prove the increasing prevalence to be a global phenomenon. However, in order to compare epidemiological data for rare autoimmune $\mathrm{AD}$, a standardized procedure of operationalization is required. With this study, we provide a differentiated approach for standardization of inclusion as well as exclusion criteria. 
Our findings emphasize the importance of a heightened clinical awareness not only to correctly diagnose the growing number of $\mathrm{AD}$ patients but also to recognize their comorbidities, especially autoimmune thyroid diseases, and also rare autoimmune diseases. Furthermore, we identify a relevant risk of adrenal crises with a quantity of about $14-17 / 100$ patient years. As every adrenal crisis is potentially life threatening and harbors the risk of recurrence, this result warrants intensified measures for prevention.

Despite the rising prevalence, the disease remains rare $(<2 / 5000)$ and thereby the medical professions need to be alerted that these patients require timely and adequate treatment and follow-up. Furthermore, elucidating the triggering factors for these epidemiological changes will lead to a better understanding of its pathophysiology and possibly new roads to immune therapy.

\section{Declaration of interest}

G Meyer, K Neumann, and K Badenhoop have nothing to declare. R Linder is employed by WINEG Scientific Institute of the Techniker Krankenkasse for Benefit and Efficiency in Health Care, which investigates the value of innovations and new programatic approaches within the Statutory Health Insurance framework.

\section{Funding}

This study was supported by the European Union 7th framework Health program FP7 with the acronym EURADRENAL under the grant agreement 2008-201167.

\section{References}

1 Arlt W \& Allolio B. Adrenal insufficiency. Lancet 2003361 1881-1893. (doi:10.1016/S0140-6736(03)13492-7)

2 Betterle C \& Morlin L. Autoimmune Addison's disease. Endocrine Development 201120 161-172. (doi:10.1159/000321239)

3 Kong MF \& Jeffcoate W. Eighty-six cases of Addison's disease. Clinical Endocrinology 199441 757-761. (doi:10.1111/j.1365-2265.1994. tb02790.x)

4 Willis AC \& Vince FP. The prevalence of Addison's disease in Coventry, UK. Postgraduate Medical Journal 199773 286-288. (doi:10.1136/ pgmj.73.859.286)

5 Laureti S, Vecchi L, Santeusanio F \& Falorni A. Is the prevalence of Addison's disease underestimated? Journal of Clinical Endocrinology and Metabolism 199984 1762. (doi:10.1210/jc.84.5.1762)

6 Lovas K \& Husebye ES. High prevalence and increasing incidence of Addison's disease in western Norway. Clinical Endocrinology 200256 787-791. (doi:10.1046/j.1365-2265.2002.t01-1-01552.x)

7 Erichsen MM, Lovas K, Skinningsrud B, Wolff AB, Undlien DE, Svarberg J, Fougner KJ, Berg TJ, Bollerslev J, Mella B et al. Clinical, immunological, and genetic features of autoimmune primary adrenal insufficiency: observations from a Norwegian registry. Journal of Clinical Endocrinology and Metabolism 200994 4882-4890. (doi:10.1210/ jc.2009-1368)
8 Bjornsdottir S, Sundstrom A, Ludvigsson JF, Blomqvist P, Kampe O \& Bensing S. Drug prescription patterns in patients with Addison's disease: a Swedish population-based cohort study. Journal of Clinical Endocrinology and Metabolism 201398 2009-2018. (doi:10.1210/ jc.2012-3561)

9 Arlt W. The approach to the adult with newly diagnosed adrenal insufficiency. Journal of Clinical Endocrinology and Metabolism 200994 1059-1067. (doi:10.1210/jc.2009-0032)

10 Hahner S, Loeffler M, Bleicken B, Drechsler C, Milovanovic D, Fassnacht M, Ventz M, Qunikler M \& Allolio B. Epidemiology of adrenal crisis in chronic adrenal insufficiency: the need for new prevention strategies. European Journal of Endocrinology 2010162 597-602. (doi:10.1530/EJE-09-0884)

11 Bleicken B, Hahner S, Ventz M \& Quinkler M. Delayed diagnosis of adrenal insufficiency is common: a cross-sectional study in 216 patients. American Journal of the Medical Sciences 2010339 525-531. (doi:10.1097/MAJ.0b013e3181db6b7a)

12 Meyer G, Hackemann A, Penna-Martinez M \& Badenhoop K. What affects the quality of life in autoimmune Addison's disease? Hormone and Metabolic Research 201345 92-95. (doi:10.1055/s-0032-1331766)

13 Riddle MS, Murray JA \& Porter CK. The incidence and risk of celiac disease in a healthy US adult population. American Journal of Gastroenterology 2012107 1248-1255. (doi:10.1038/ajg.2012.130)

14 Berhan Y, Waernbaum I, Lind T, Mollsten A \& Dahlquist G. Thirty years of prospective nationwide incidence of childhood type 1 diabetes: the accelerating increase by time tends to level off in Sweden. Diabetes 2011 60 577-581. (doi:10.2337/db10-0813)

15 Bruno G, Maule M, Merletti F, Novelli G, Falorni A, Iannilli L, Altobelli E d'Annunzi G, Piffer S, Pozzilli P et al. Age-period-cohort analysis of 1990-2003 incidence time trends of childhood diabetes in Italy: the RIDI study. Diabetes 201059 2281-2287. (doi:10.2337/db10-0151)

16 Bruno G, Maule M, Biggeri A, Ledda A, Mannu C, Merletti F \& Songini M. More than twenty years of registration of type 1 diabetes in Sardinian children: temporal variations of incidence with age, period of diagnosis and year of birth. Diabetes 201362 3542-3546. (doi:10.2337/ db12-1771)

17 Dahlquist GG, Nystrom L \& Patterson CC. Incidence of type 1 diabetes in Sweden among individuals aged 0-34 years, 1983-2007: an analysis of time trends. Diabetes Care 201134 1754-1759. (doi:10.2337/ dc11-0056)

18 Sipetic S, Maksimovic J, Vlajinac H, Ratkov I, Sajic S, Zdravkovic D \& Sipetic T. Rising incidence of type 1 diabetes in Belgrade children age $0-14$ in the period from 1982 to 2005. Journal of Endocrinological Investigation 201236 307-312. (doi:10.3275/8619)

19 Krokki O, Bloigu R, Reunanen M \& Remes AM. Increasing incidence of multiple sclerosis in women in Northern Finland. Multiple Sclerosis 2011 17 133-138. (doi:10.1177/1352458510384012)

20 Husebye E \& Lovas K. Pathogenesis of primary adrenal insufficiency. Best Practice \& Research. Clinical Endocrinology \& Metabolism 200923 147-157. (doi:10.1016/j.beem.2008.09.004)

21 Kahles H, Morahan G, Todd JA \& Badenhoop K. Association analyses of the vitamin D receptor gene in 1654 families with type I diabetes. Genes and Immunity 200910 (Suppl 1) S60-S63. (doi:10.1038/gene.2009.93)

22 Lopez ER, Zwermann O, Segni M, Meyer G, Reincke M, Seissler J, Herwig J, Usadel KH \& Badenhoop K. A promoter polymorphism of the CYP27B1 gene is associated with Addison's disease, Hashimoto's thyroiditis, Graves' disease and type 1 diabetes mellitus in Germans. European Journal of Endocrinology 2004151 193-197. (doi:10.1530/ eje.0.1510193)

23 Munger KL, Levin LI, Massa J, Horst R, Orban T \& Ascherio A. Preclinical serum 25-hydroxyvitamin $\mathrm{D}$ levels and risk of type 1 diabetes in a cohort of US military personnel. American Journal of Epidemiology 2013 177 411-419. (doi:10.1093/aje/kws243)

24 Zhang HL \& Wu J. Role of vitamin D in immune responses and autoimmune diseases, with emphasis on its role in multiple sclerosis. Neuroscience Bulletin 201026 445-454. (doi:10.1007/s12264-010-0731-8) 
25 Langer J, Penna-Martinez M, Wallasch M, Bon D \& Badenhoop K. Time trend of UVB irradiation and the vitamin D concentration in German type 1 diabetes patients. Journal of Steroid Biochemistry and Molecular Biology 2013136 218-220. (doi:10.1016/j.jsbmb.2012.11.008)

26 Javierre BM, Hernando H \& Ballestar E. Environmental triggers and epigenetic deregulation in autoimmune disease. Discovery Medicine $201112535-545$

27 Miller FW, Alfredsson L, Costenbader KH, Kamen DL, Nelson LM, Norris JM \& De Roos AJ. Epidemiology of environmental exposures and human autoimmune diseases: findings from a National Institute of Environmental Health Sciences Expert Panel Workshop. Journal of Autoimmunity 201239 259-271. (doi:10.1016/ j.jaut.2012.05.002)

28 Hughes AM, Lucas RM, McMichael AJ, Dwyer T, Pender MP, Van der Mei I, Taylor BV, Valery P, Chapman C, Coulthard A et al. Early-life hygiene-related factors affect risk of central nervous system demyelination and asthma differentially. Clinical and Experimental Immunology 2013172 466-474. (doi:10.1111/cei.12077)

Received 17 September 2013

Revised version received 21 November 2013

Accepted 9 December 2013 